\title{
Clinical Results of Direct Arthroscopic Excision of Popliteal Cyst Using a Posteromedial Portal
}

\author{
Jin Ho Cho, $\mathrm{MD}, \mathrm{PhD}$ \\ Department of Orthopedic Surgery, Ilsan Paik Hospital, Inje University College of Medicine, Goyang, Korea
}

Purpose: To evaluate the efficacy of the direct arthroscopic excision of a popliteal cyst without additional skin incision using a posteromedial portal based on minimum 2 year follow-up clinical results.

Materials and Methods: From January 2003 to January 2010, 105 patients (111 cases) with popliteal cyst have been treated by a direct arthroscopic excision. Direct arthroscopic excision using a 70 degree arthroscopy and posteromedial portal can correct the valvular mechanism of capsular fold and reduce the complications with no additional skin incision at the popliteal area. In all cases, preoperative magnetic resonance imaging (MRI) was performed to detect combined intraarticular pathology. At 2 years postoperatively, a follow-up ultrasonography or MRI was performed to detect the recurrence of cysts. We used Rauschning and Lindgren criteria for clinical evaluation.

Results: All cases had neither recurrence nor complaints of pain, swelling, or functional impairment more than 2 years after the surgery. At ultrasonography or MRI, no recurrence was found, and 5 complications were 1 skin lesion and 4 hematoma. Postoperatively, Rauschning and Lindgren criteria were more than grade 1.

Conclusions: Direct arthroscopic excision using 70 degree arthroscope and posteromedial portal is an effective method for the treatment of popliteal cyst.

Key words: Popliteal cyst, Arthroscopy, Posteromedial portal, Excision, Clinical results.

\section{Introduction}

The preferred treatment methods for popliteal cysts have been conservative. As for the symptomatic cysts, open resection has been preferred. However, open resection has resulted in higher recurrence and more frequent cosmetic problems ${ }^{1,2)}$. Studies of the pathogenesis of popliteal cysts have shown that they are associated with intraarticular pathology and valvular mechanism $^{3,4)}$, thus, it implies that the correction of the valvular

Received May 4, 2012; Revised (1st) June 4, 2012; (2nd) July 6, 2012;

(3rd) July 16, 2012; Accepted August 16, 2012.

Correspondence to: Jin Ho Cho, MD.

Department of Orthopedic Surgery, Ilsan Paik Hospital, Inje University

College of Medicine, 170 Juhwa-ro, Ilsanseo-gu, Goyang 411-706, Korea. Tel: +82-31-910-9733, Fax: +82-31-910-7967

Email: osd07@paik.ac.kr

This is an Open Access article distributed under the terms of the Creative Commons Attribution Non-Commercial License (http://creativecommons.org/licenses/by-nc/3.0/) which permits unrestricted non-commercial use, distribution, and reproduction in any medium, provided the original work is properly cited. mechanism can prevent the recurrence of cysts. With recent advances in arthroscopic techniques, arthroscopic treatment for popliteal cyst could be effective for both cysts and associated intra-articular pathology $y^{3,5)}$. The purpose of this study was to evaluate the clinical results of direct arthroscopic excision of popliteal cyst using a posteromedial portal and 70 degree arthroscope without additional skin incision.

\section{Materials and Methods}

From January 2003 to January 2010, 111 cases of 105 patients with popliteal cysts were treated arthroscopically. The patients included 24 men and 81 women. The average age was 57 years (range, 30 to 80 years). The popliteal cysts were located in the right knee in 60 cases, in the left knee in 45 cases, and in both knees in 6 cases. To evaluate the intra-articular lesions, preoperative magnetic resonance imaging (MRI) was performed in all patients (Fig. 1). At 2 years postoperatively, ultrasonography or MRI was performed again to detect the recurrence.

The main complaint of the popliteal cysts consists of popliteal pain and discomfort. Patients underwent conservative treatment for about 2.8 years. Of them, 67 patients underwent aspiration of 

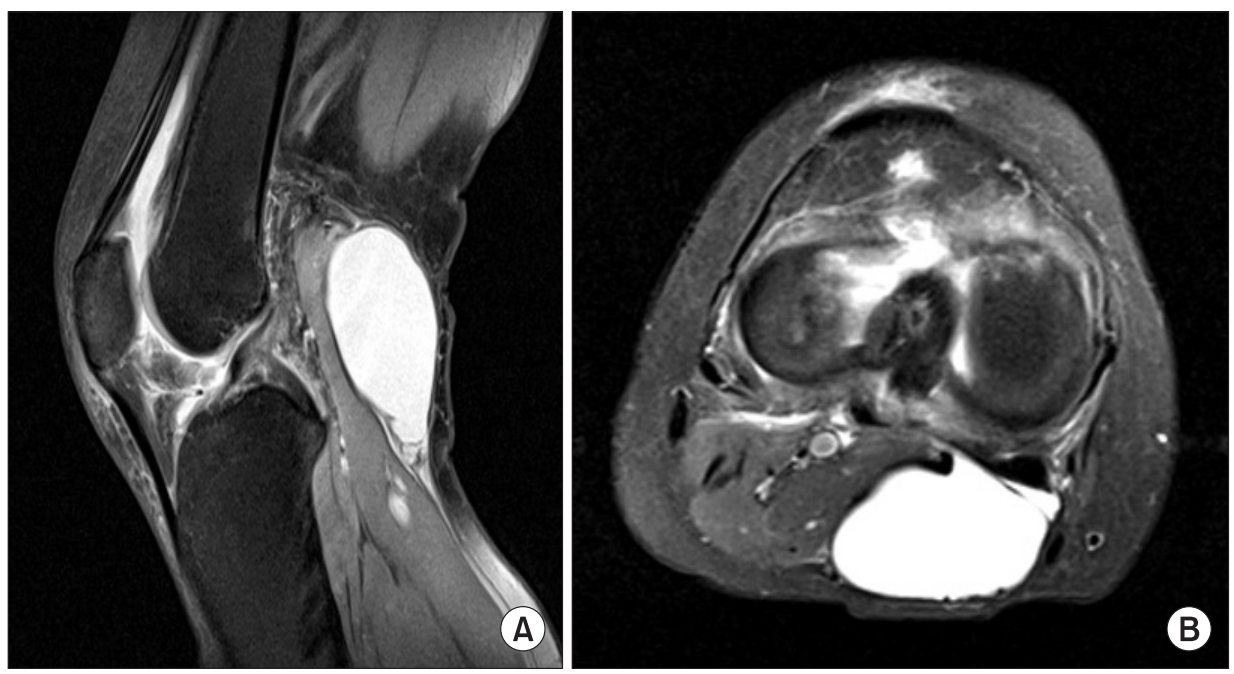

Fig. 1. Large popliteal cyst detected in (A) sagittal T2 and (B) axial view of magnetic resonance imaging on the right knee.
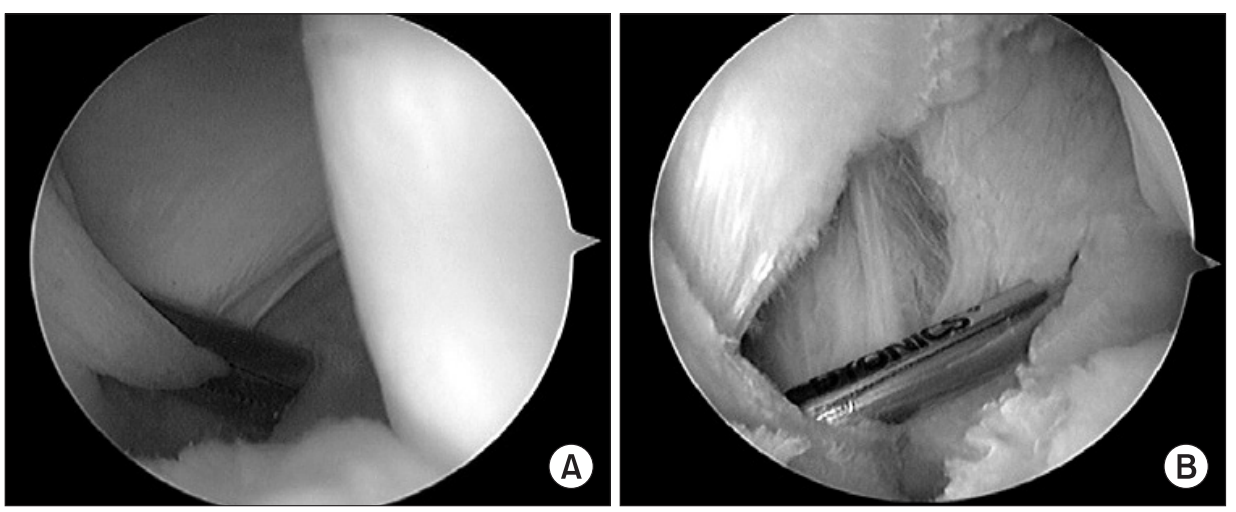

Fig. 2. (A) An arthroscopic view shows the transverse band with probe inserted via posteromedial portal on the right knee. (B) The opening between popliteal cyst and joint space can be seen on the right knee.

cysts before visiting our hospital.

The criteria for the operative treatment included an MRI detected cystic lesion accompanied by symptoms associated with an intra-articular lesion, recurrent popliteal cysts after aspiration, and mass-like symptoms such as swelling, pain and limitation of motion in the knee joint.

\section{Surgical Technique}

The patients underwent arthroscopic surgery under general or spinal anesthesia in the supine position. Routine arthroscopic examination of the knee joint was performed through the standard anterolateral portal with 30 degree arthroscopy. Having examined the intra-articular pathologies in the knee joint, we fixed the arthroscopy shell, removed the arthroscopy and reinserted the closing rod. The arthroscope was redirected toward the posteromedial compartment from the anterolateral portal through the intercondylar notch with the knee at 60 degree flexion. We examined the presence of the capsular fold in the posteromedial wall by 30 degree arthroscopy and proceeded with inserting the spinal needle into the borderline which was composed of the medial collateral ligament (MCL), medial gastrocnemius and semimembranous with knee at 90 degree flexion. While inserting the needle, the knee joint was visualized by transillumination to avoid the vessel. If the position of the needle is proper, we incised the skin and made a posteromedial portal. Maintaining the arthroscopic field by 30 degree arthroscopy, a probe was inserted to find the connection opening to the cyst. We resected the capsular fold by using the shaver, arthrocare and arthroscopic scissor (Fig. 2). After excision of the capsular fold, 30 degree arthroscope was advanced into the cystic wall. To obtain a wider field of cystic inner cavity, we changed the 70 degree arthroscopy to obtain a wider operative field (Fig. 3). We kept a 70 degree arthroscopy via an anterolateral portal and an arthroscopic shaver was inserted into the cystic wall via the posteromedial portal (Fig. 4), Avoiding the tendon, vessel and nerve injury, we removed the materials like the loose fragment and band in the cystic wall completely (Fig. 5). Associated intraarticular disorders, such as tears of the medial meniscus, chondral 

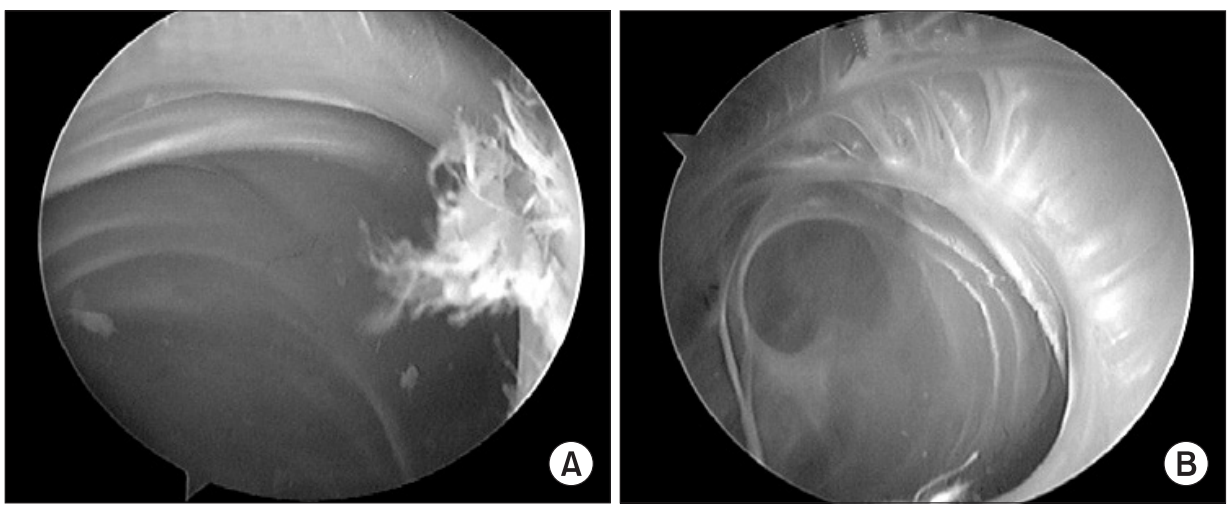

Fig. 3. (A) We can see the partial cystic wall within a cyst via 30 degree arthroscopy, (B) however, we can see more wide cystic wall within cyst via 70 degree arthroscopy.
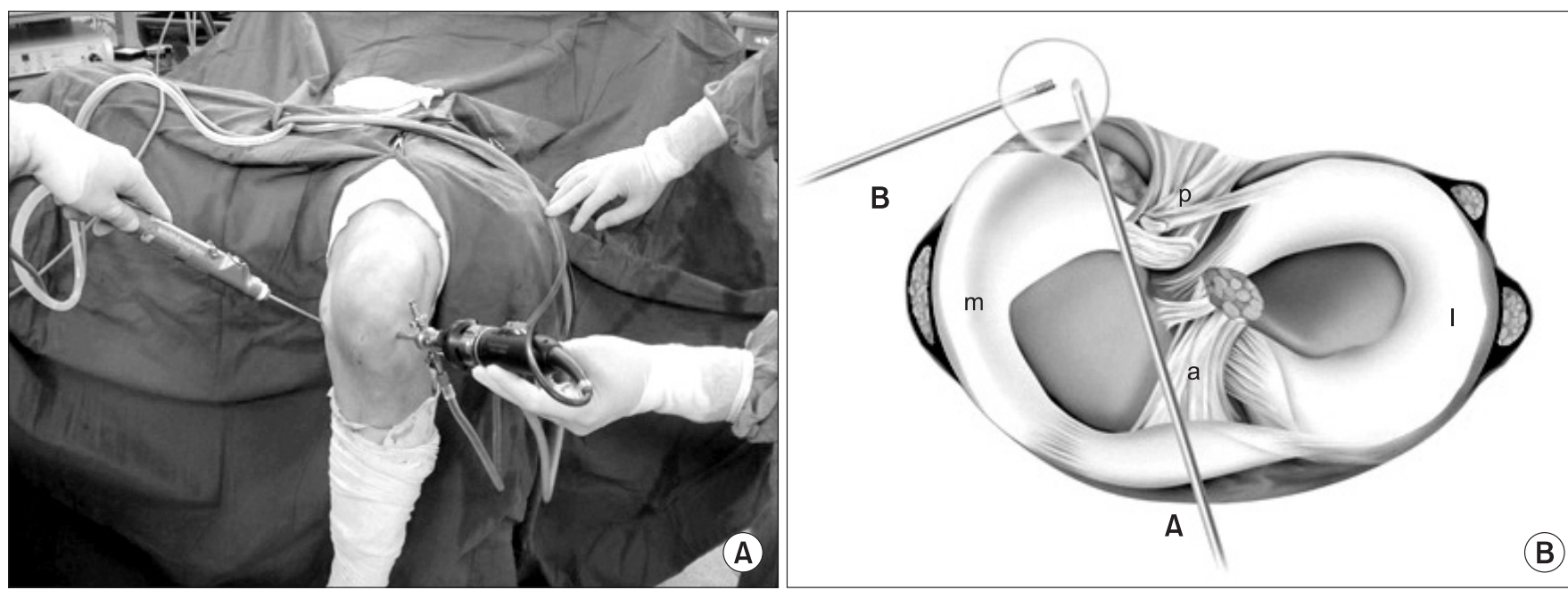

Fig. 4. (A) Operative photography. (B) Schematic drawing of 70 degree arthroscopy is introduced within popliteal cyst via anterolateral portal, and then arthroscopic shaver is introduced within popliteal cyst via posteromedial portal on the left knee. m: medial meniscus, l: lateral meniscus, a: anterior cruciate ligament, p: posterior cruciate ligament.
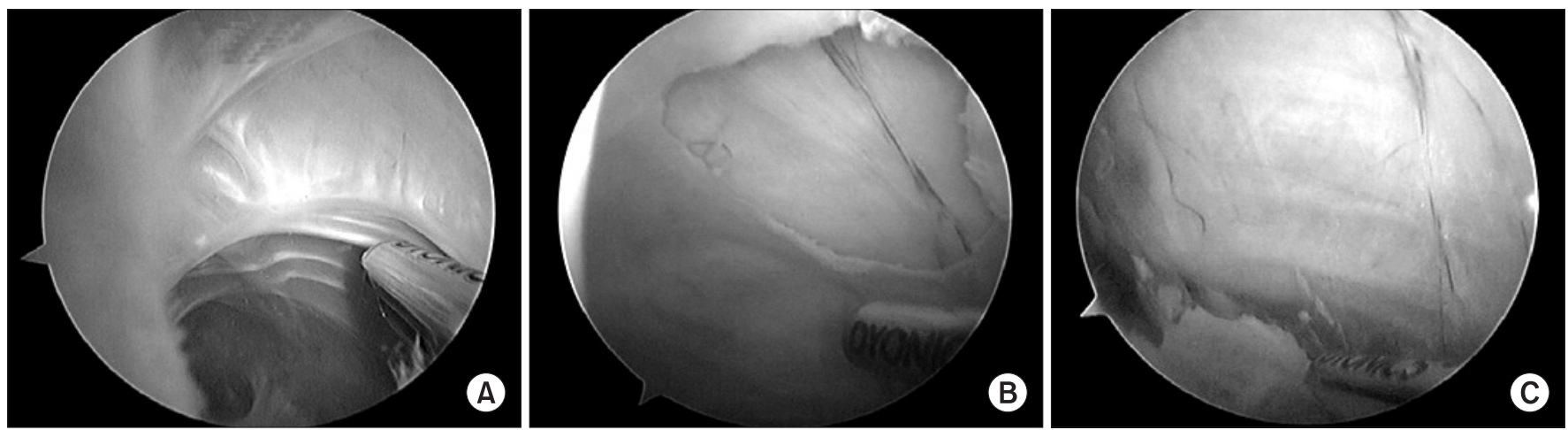

Fig. 5. (A, B) We can excise cystic wall inner side with shaver and can avoid subcutaneous capillary vessels injury via 70 degree arthroscopic view. (C) Arthroscopic view show complete cystic excision on the right knee. All procedures were done within cyst without additional skin incision.

lesions, and synovitis were then treated via the arthroscopic procedure. Once a hemo-vac drain was inserted into the space by posteromedial portal, a compressive dressing was applied.
Operation time was from 20 to 40 minutes depending on the combined injury. 


\section{Postoperative Treatment}

We removed $\mathrm{H}$-vac drain 2 days after surgery. Then, partial weight bearing with crutch and active-passive motion was started. Full weight bearing was permitted from 1 week after surgery in patients who had not been treated by microfracture technique.

\section{Evaluation}

In all cases, preoperative MRI was performed to detect a combined intraarticular pathology. At 2 year postoperatively, follow-up ultrasonography (77\%) or MRI (23\%) was performed to detect the recurrence of cysts. We used Rauschning and Lindgren criteria for clinical evaluation.

\section{Results}

Meniscal lesions were most commonly found in 67 cases (60.3\%), 52 of which were medial meniscus tears. The type of tear includes degenerative horizontal tear in 44 cases and complex

Table 1. Intraarticular Knee Pathologies Associated with Popliteal Cyst

\begin{tabular}{lc}
\hline \multicolumn{1}{c}{ Pathology } & No. of cases $(\%)$ \\
\hline Medial meniscus tear & $67(60)$ \\
Degenerative change & $44(40)$ \\
Synovitis and synovial hypertrophy & $26(23)$ \\
Chondromalacia patellae & $5(5)$ \\
Plica syndrome & $4(4)$ \\
Loose body (intra-articular) & $1(1)$ \\
\hline
\end{tabular}

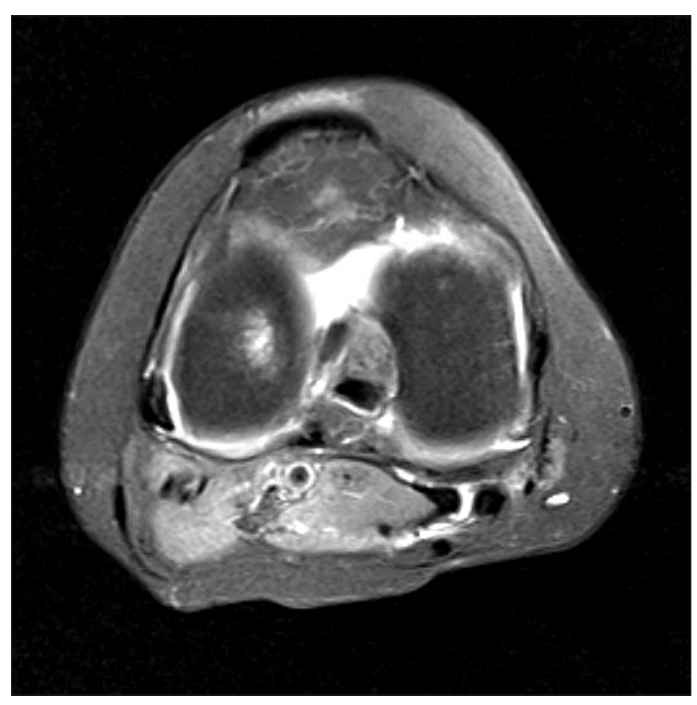

Fig. 6. At 2 year after surgery, a follow-up axial view magnetic resonance imaging show elimination of the popliteal cyst on the right knee. tear in 5 cases. In the other cases, we discovered degenerative osteoarthritis in 44 cases (39.6\%), synovitis and synovial hypertrophy in 26 cases $(23.4 \%)$, chondromalacia and cartilage defect of patellofemoral joint in 5 cases $(4.5 \%)$, plica syndrome in 4 cases $(3.6 \%)$ and loose body in 1 case (1\%) (Table 1 ).

We found the transverse capsular fold in all the patients and the type of posteromedial wall according to Johnson classification ${ }^{6}$ was type 1 in 12 cases, type 2 in 80 cases and type 3 in 19 cases. After 2 years, all cysts were not palpated and neither could we find the recurrence of cysts on a postoperative MRI (Fig. 6). No one complained about pain or limitation in daily activities. Functional evaluation was performed using Rauschning and Lindgren $^{7)}$ criteria. On the clinical evaluation, all patients ended up with grade 1 (Table 2). One patient had a 1 degree burn injury at the posteromedial portal site by a shaver and 4 patients had hematoma formation, but this was resolved after compression and rest.

\section{Discussion}

The principle finding of this study was that 70 degree arthroscopy improved visual field within the popliteal cyst so that we could excise the cyst wall completely, which resulted in reduced recurrence rates. Although the operative techniques of popliteal cysts differ from author to author ${ }^{8)}$, arthroscopic resection is being actively attempted, as of recently ${ }^{9)}$. The popliteal cyst is almost never an isolated pathology in an adult knee. An open surgical excision cannot be considered as a definitive solution in most patients. We believe that addressing the opening or rather the connection between the joint cavity and cyst is a key procedure for completely excising the cyst. Arthroscopic procedures have several benefits. They are relatively simple, allow early rehabilitation, require minimal incision, and effectively address any concomitant intraarticular pathologies, and most importantly, they can remove the opening of the $\mathrm{cyst}^{10)}$. The frequency of recurrence, popliteal pain and limitation of motion after simple surgical resection ${ }^{11,12)}$, as well as intraoperative

Table 2. Clinical Results of Popliteal Cyst Excision (Rauschning and Lindgren)

\begin{tabular}{lcc}
\hline & Before surgery & After 2 year \\
\hline Grade 0 & 0 & 98 \\
Grade 1 & 96 & 13 \\
Grade 2 & 10 & 0 \\
Grade 3 & 5 & 0 \\
\hline
\end{tabular}

Values are presented as number of cases. 
position change following the simple surgical resection, lead to such change in the therapeutic strategy.

Reportedly, the causes of popliteal cyst include trauma, osteoarthritis $^{13)}$, rheumatoid arthritis ${ }^{2,14,15)}$, meniscal tear ${ }^{5,16)}$, and loose body. Johnson et al. ${ }^{6}$ described the frequency of injury in the medial meniscus tear as $68 \%$, in osteoarthritis as $81 \%$, in loose body as $38 \%$, in edema as $35 \%$, and in cartilage injury of the patellofemoral joint as $30 \%$. In our study, medial meniscal tear and osteoarthritis occurred respectively at $47 \%$ and $40 \%$ frequency, similar to that of Johnson.

With an understanding of pathogenesis that a valvular mechanism of capsular fold and continuous unidirectional flow from the joint cavity to the cyst, many authors noted that intraarticular joint pathology must be corrected during the surgery for reducing the recurrence ${ }^{3,5)}$. To prevent the recurrence of cyst, Sansone and De Ponti ${ }^{4}$ reported the importance of removal of the valve and unidirectional flow. Johnson et al. ${ }^{6)}$ typed cysts according to the anatomic structure of the posteromedial capsular wall. Type III has a transverse band and a comminuted hole. In the past, as a method of treating this hole, Hughston et al..$^{17)}$ performed a simple capsular suture, Childress ${ }^{16)}$ described an augmentation using the tendon of the gastrocnemius and semimembranous. Rauschning ${ }^{12)}$ performed a pedicled graft of the medial head of the gastrocnemius. However, in this method, the gastrocnemius and semimembranosus are incorporated together and the split is difficult. This can prevent precise capsular suture, and the suture site can rupture because of the increased pressure during the flexion and extension exercise of the knee joint ${ }^{14)}$.

Rauschning and Lindgren ${ }^{7)}$ described that the correction of joint pathology for removal of the unidirectional flow was more important than the methods of the operative techniques. Lindgren and Willen ${ }^{18)}$ noted that in about $50 \%$ of normal adults, a connection exists between the knee joint cavity and the gastrocnemius-semimembranosus bursa, even without a popliteal cyst. Sansone and De Ponti ${ }^{4)}$ reported that no weakness of the joint structure and no complications occur despite an enlargement of the posteromedial capsular hole. Ahn and Ko ${ }^{9,11}$ reported that the enlargement of the posteromedial connecting hole of about $5 \mathrm{~mm}$ could converse the unidirectional flow to the bidirectional flow. If any shift of the fluid occurs between the joint and the cyst, spontaneous reabsorption occurs because of the bidirectional flow.

Our technique is arthroscopic direct cystic excision using a 70 degree arthroscopy to obtain a more accurate operative field and posteromedial portal for resection of capsular fold and cystic wall by shaver and scissors. Our technique is different from that of Ahn's, ${ }^{7,818}$ in the absence of additional skin incision for the insertion of the shaver, removal of a cystic wall and detect the capsular fold in the posteromedial wall while enlarging the opening. All cases had no recurrence and excellent functional outcomes more than 2 year after surgery.

This study has a few limitations. First, there was no comparison group. Second, we focused on the cyst recurrence. Therefore, we did not evaluate the various scores for clinical results.

\section{Conclusions}

Direct arthroscopic excision using 70 degree arthroscopy and posteromedial portal can correct the valvular mechanism of capsular fold and reduce the complications with no additional skin incision at the popliteal area. We have experienced no recurrence and excellent clinical results at the minimum 2 year follow-up.

\section{References}

1. Cho DY, Sec JG, Haam YG. The management of Baker's cyst (the new surgical technique for the prevention of recurrence). J Korean Orthop Assoc. 1994;29:288-93.

2. Kanekasu K, Nagashima K, Yamauchi D, Yamakado K. A clinical study of arthroscopic cystectomy on popliteal cysts associated with rheumatoid arthritis. Ryumachi. 1997;37:761-9.

3. Lindgren PG. Gastrocnemio-semimembranosus bursa and its relation to the knee joint. III. Pressure measurements in joint and bursa. Acta Radiol Diagn (Stockh). 1978;19:37788.

4. Sansone V, De Ponti A. Arthroscopic treatment of popliteal cyst and associated intra-articular knee disorders in adults. Arthroscopy. 1999;15:368-72.

5. Smith JT, Yandow SM. Benign soft-tissue lesions in children. Orthop Clin North Am. 1996;27:645-54.

6. Johnson LL, van Dyk GE, Johnson CA, Bays BM, Gully SM. The popliteal bursa (Baker's cyst): an arthroscopic perspective and the epidemiology. Arthroscopy. 1997;13:6672.

7. Rauschning W, Lindgren PG. Popliteal cysts (Baker's cysts) in adults. I. Clinical and roentgenological results of operative excision. Acta Orthop Scand. 1979;50:583-91.

8. Vahvanen V. Popliteal cyst. A follow-up study on 42 operatively treated patients. Acta Orthop Scand. 1973;44:303-10. 
9. Ahn JH, Ko SH. Arthroscopic shaving cystectomy of popliteal cyst. J Korean Orthop Assoc. 2002;37:364-8.

10. Ahn JH, Yoo JC, Lee SH, Lee YS. Arthroscopic cystectomy for popliteal cysts through the posteromedial cystic portal. Arthroscopy. 2007;23:559.e1-4.

11. Ko S, Ahn J. Popliteal cystoscopic excisional debridement and removal of capsular fold of valvular mechanism of large recurrent popliteal cyst. Arthroscopy. 2004;20:37-44.

12. Rauschning W. Popliteal cysts (Baker's cysts) in adults. II. Capsuloplasty with and without a pedicle graft. Acta Orthop Scand. 1980;51:547-55.

13. Gristina AG, Wilson PD. Popliteal cysts in adults and children. A review of 90 cases. Arch Surg. 1964;88:357-63.

14. Fujikawa K. Arthrographic study of the rheumatoid knee.
Part 1. Synovial proliferation. Ann Rheum Dis. 1981;40:33243.

15. Moore CP, Sarti DA, Louie JS. Ultrasonographic demonstration of popliteal cysts in rheumatoid arthritis. A noninvasive technique. Arthritis Rheum. 1975;18:577-80.

16. Childress HM. Popliteal cysts associated with undiagnosed posterior lesions of the medial meniscus. The significance of age in diagnosis and treatment. J Bone Joint Surg Am. 1970;52:1487-92.

17. Hughston JC, Baker CL, Mello W. Popliteal cyst: a surgical approach. Orthopedics. 1991;14:147-50.

18. Lindgren PG, Willen R. Gastrocnemio-semimembranosus bursa and its relation to the knee joint. I. Anatomy and histology. Acta Radiol Diagn (Stockh). 1977;18:497-512. 